\title{
An Acid-Base Controllable Hierarchical Nanostructure from a NIR- Absorbing Conjugated Polyrotaxane-Based Optical Molecular Switch
}

\author{
Mandapati V. Ramakrishnam Raju, ${ }^{\dagger}$ P. Raghunath, ${ }^{\ddagger}$ Ming-Chang Lin, ${ }^{\dagger}$ and Hong-Cheu Lin* ${ }^{\dagger}$ \\ ${ }^{\dagger}$ Department of Materials Science and Engineering, National Chiao Tung University, Hsinchu 300, Taiwan \\ ${ }^{\ddagger}$ Center for Interdisciplinary Molecular Science, Department of Applied Chemistry, National Chiao Tung University, Hsinchu 300, \\ Taiwan
}

Supporting Information

ABSTRACT: To investigate the supramolecular interactions of the mechanically interlocked rotaxane pendants and conjugated polymer backbones, four analogous polymers were systematically synthesized by copolymerization of a 9alkylidene- $9 H$-fluorene monomer with various monomers, which contained a diketopyrrolopyrrole unit tethered with a dumbbell unit, a metalated [2] rotaxane, a demetalated orthogonal H-bonded [2] rotaxane, and a simple alkyl chain, to furnish P1, P2, P3, and P4, respectively. Prevailing ${ }^{1} \mathrm{H}$ NMR and UV-vis to NIR titration profiles indicated that the novel

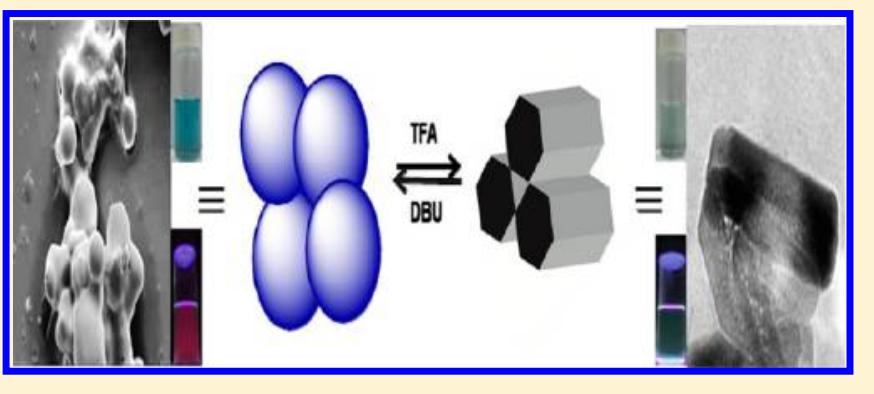
polyrotaxane P3 showed a sensitive and reversible acid-base molecular switch capability via supramolecular interactions in contrast to the other polymers (P1, P2, and P4). Compared with the other polymers, P3 possessed a narrower bandgap, which was also confirmed by the computational study. Prominently, the monitoring of a controllable nanoself-assembly process of P3 was obtained by reversible acid-base molecular switch approaches. The orthogonal H-bonded pendant [2] rotaxane unit and the steric demand of P3 judiciously allowed to morph into a hierarchical nanostructure via interconvertible $\mathrm{H}$-bonds, anion $-\pi$ and $\pi-\pi$ stackings, and hydrophobic interactions.

\section{INTRODUCTION}

The quest for developing novel and creative mechanically interlocked polymeric architectures (MIPAs) stimulated us, since these architectures had been found potential applications in fields like nanoelectromechanical systems (NEMS), ${ }^{1}$ insulated molecular wires (IMWs), ${ }^{2}$ and controlled drug release. ${ }^{3}$ During the past two decades, rotaxanes and catenanes have been experienced revolutionary changes in their synthetic protocols; ${ }^{4}$ thus, their convenient and modular fashioned approaches allowed to realize complex macromolecular architectures. In this context, conjugated polymers with mechanically interlocked units in either main chains ${ }^{5}$ or side chains ${ }^{6}$ have drawn a special attention among materials and supramolecular chemists. Unique optical and semiconducting properties of $\pi$-conjugated polymers have long been motivated to construct novel optoelectronic devices. ${ }^{7}$ In addition to this molecular self-assembly properties of copolymers, $\pi$-conjugated structures have created a new dimension for these polymers. ${ }^{8}$

Supramolecular complex architectures, such as polyrotaxanes, polycatenanes, and daisy chain polymers, have often been offering unique properties in contrast to their single ensemble counterparts. ${ }^{9}$ Enormous strides have long been made in the field of MIPAs to the realization of precise molecular mechanics in imparting the unusual photophysical properties to the $\pi$ conjugated polymers at the molecular level. Swager and coworkers have reported how the optical properties of conjugated main-chain polymers were affected by the rotaxane exciplex. ${ }^{10}$
Stoddart and co-workers have extensively investigated bistable MIPAs and developed molecular electronic memory devices with cross sections even smaller than a white blood cell. ${ }^{11}$ They suggested that the unique degrees of freedom and controlled molecular motions of mechanical bonds altered the photophysical properties of MIPAs; however, most of these reports were associated with macrocycle units, such as cyclodextrins, ${ }^{12}$ cucurbiturils, ${ }^{13}$ and crown ethers. ${ }^{14}$ Nevertheless, there is no such example of polyrotaxane architecture containing an orthogonal H-bonded macrocycle unit.

Self-assembled bilayer membrane nanostructures of phospholipids have been elegantly utilized by the nature to compartmentalize vital biological reactions, inspired by many artificial synthetic amphiphilic receptors to be developed for specific drug delivery ${ }^{15}$ and ion-channel transportation. ${ }^{16}$ The nanostructures and their morphological changes were dependent on individual intermolecular interactions which were controllable by the concentrations, polarities, $\mathrm{pH}$ values, functional groups, and geometries of the units. Thus, the remarkable stimuli responsiveness of these nanostructures combined with switchable rotaxane units makes them expedient candidates in designing novel and functional MIPAs. ${ }^{1 \mathrm{~b}, 8 \mathrm{~b}}$ Moreover, synthetic amphiphilic receptors possessing extended

Received: July 15, 2013

Revised: August 12, 2013

Published: August 23, 2013 
Scheme 1. General Polymerization Route of the Side-Chain MIPAs P2 and P3 and Control Polymers P1 and P4

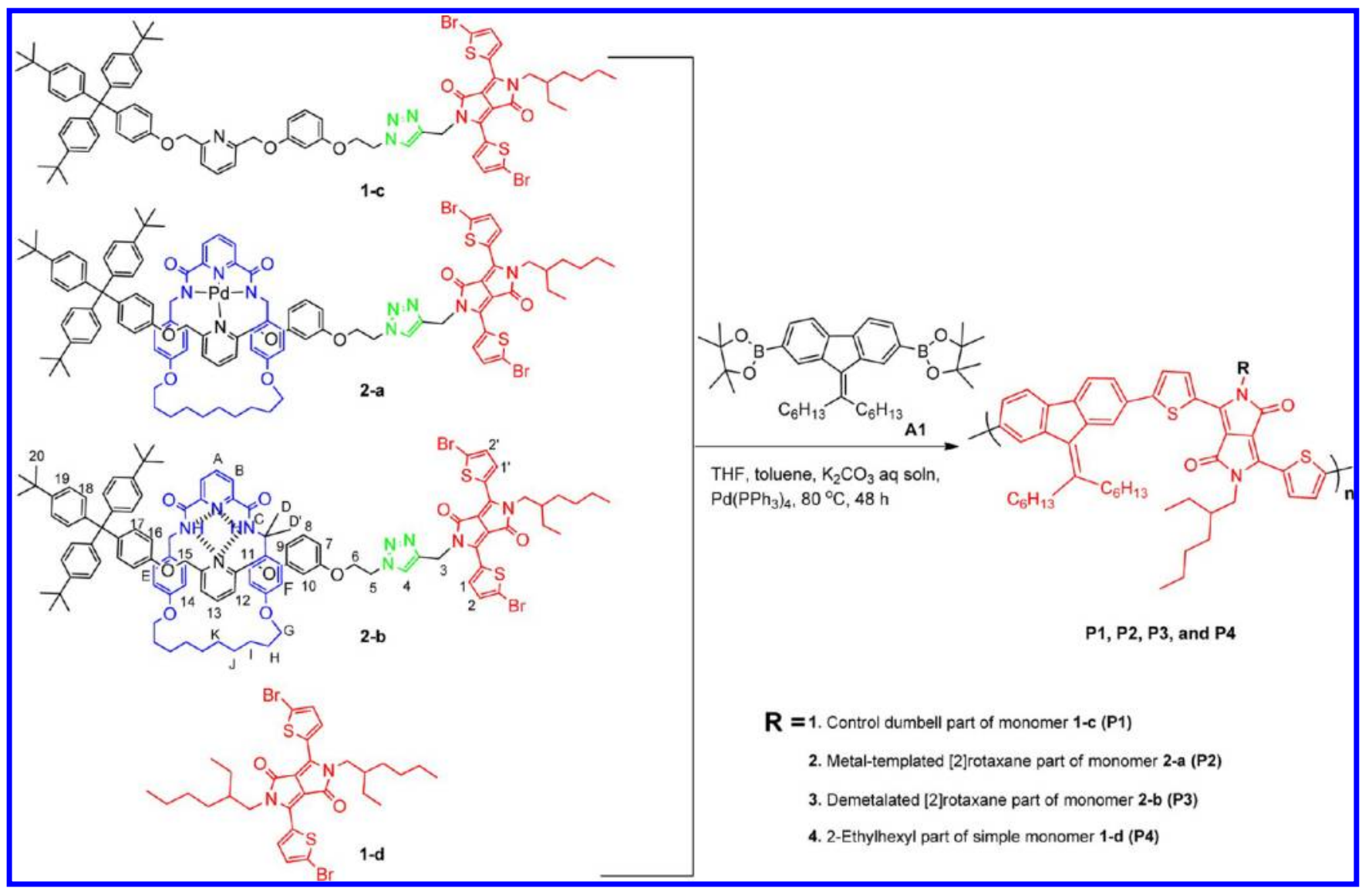

conjugations close to the infrared region are highly demanding, as this region provides the diminished Rayleigh-Tyndall scattering of light which is suitable for most of the biological specimen imaging. ${ }^{17}$

From the viewpoint of creating novel hierarchical nanostructures, the orthogonal hydrogen-bonded [2]rotaxane units in conjunction with polymeric structures might provide interesting MIPAs. However, to the best of our knowledge, such systems have not been investigated previously. To mimic this, we need a bottom-up approach with an amphiphilic synthetic receptor that possesses a unique topologically controlled $\mathrm{H}$ bonded macrocycle unit tethered to an extended $\pi$-conjugated planar polymeric backbone. We have recently developed a novel diketopyrrolopyrrole-based [2] rotaxane architecture with an orthogonal $\mathrm{H}$-bonded macrocycle unit, which showed remarkable stimuli responsiveness and selective anion sensing capability. ${ }^{18}$ With the aim of utilizing unique topological cavities of MIPAs in stimuli-controlled macromolecular selfassembled nanostructures, which could not be attained previously, we extended our efforts to create novel and functional MIPAs and to study the effects of rotaxanation on the thermal and optical properties as well as the self-assembly process of MIPAs.

In this study, we synthesized a series of new polymers by copolymerizing the 9-alkylidiene-9H-fluorene (A1) monomer unit with various monomers, including the diketopyrrolopyrrole tethered with dumbbell (1-c), metalated [2] rotaxane (2-a), demetalated [2] rotaxane (2-b), and simple alkyl chain monomers (1-d), by Suzuki coupling strategy to afford copolymers P1, P2, P3, and P4, respectively, as shown in Scheme 1 . We employed these new MIPAs in the host-guest chemistry, in which orthogonal H-bonded pendant [2]rotaxanated P3 showed a selective and reversible optical response toward the trifluoroacetic acid (TFA) in comparison with the other polymers (P1, P2, and P4). We explored the effects of a topological H-bonding on the optical and selfassembled properties of the $\pi$-conjugated main-chain polymer P3. Furthermore, we carried out computational density functional theory (DFT) and Parameterized Model number 3 (PM3) calculations to understand the basic molecular interactions and electron absorption properties of MIPAs. Macromolecular self-assembled nanostructures were examined by using various microscopic analysis (AFM, SEM, and TEM) and dynamic light scattering (DLS) studies. Herein, we provide a novel and yet a simple paradigm of MIPAs that showed a sensitive controllable and reversible acid-base sensing as well as molecular self-assembly properties.

\section{EXPERIMENTAL SECTION}

Sample Preparation. Stock solutions of polymers, monomers (10 $\mu \mathrm{M})$, and TFA $(10 \mathrm{mM})$ were prepared (using freshly distilled and degassed solvents, further diluted to their desired volumes before experiments). Polymer solutions were spin-coated at $1500 \mathrm{rpm}$ on $2 \times$ $2 \mathrm{~cm}^{2}$ glass plates for solid state UV-vis to NIR absorption measurements. Solutions were sonicated and filtered through fine micrometer filter prior to dynamic light scattering analysis. For microscopic experiments, such as AFM and FE-SEM, freshly prepared solutions were drop-cast on $1 \times 1 \mathrm{~cm}^{2} \mathrm{SiO}_{2} / \mathrm{Si}$ substrates, and gold coatings were used in FE-SEM sample analysis. Furthermore, the samples were drop-cast on carbon-coated $\mathrm{Cu}$ grids in TEM analysis. All samples were evaporated in a vacuum oven prior to analysis.

Calculation Methods. We carried out density functional theory (DFT) calculations to elucidate the complex nature of interaction in 
Scheme 2. Chemical Structures of Polymers P1, P2, P3, and P4

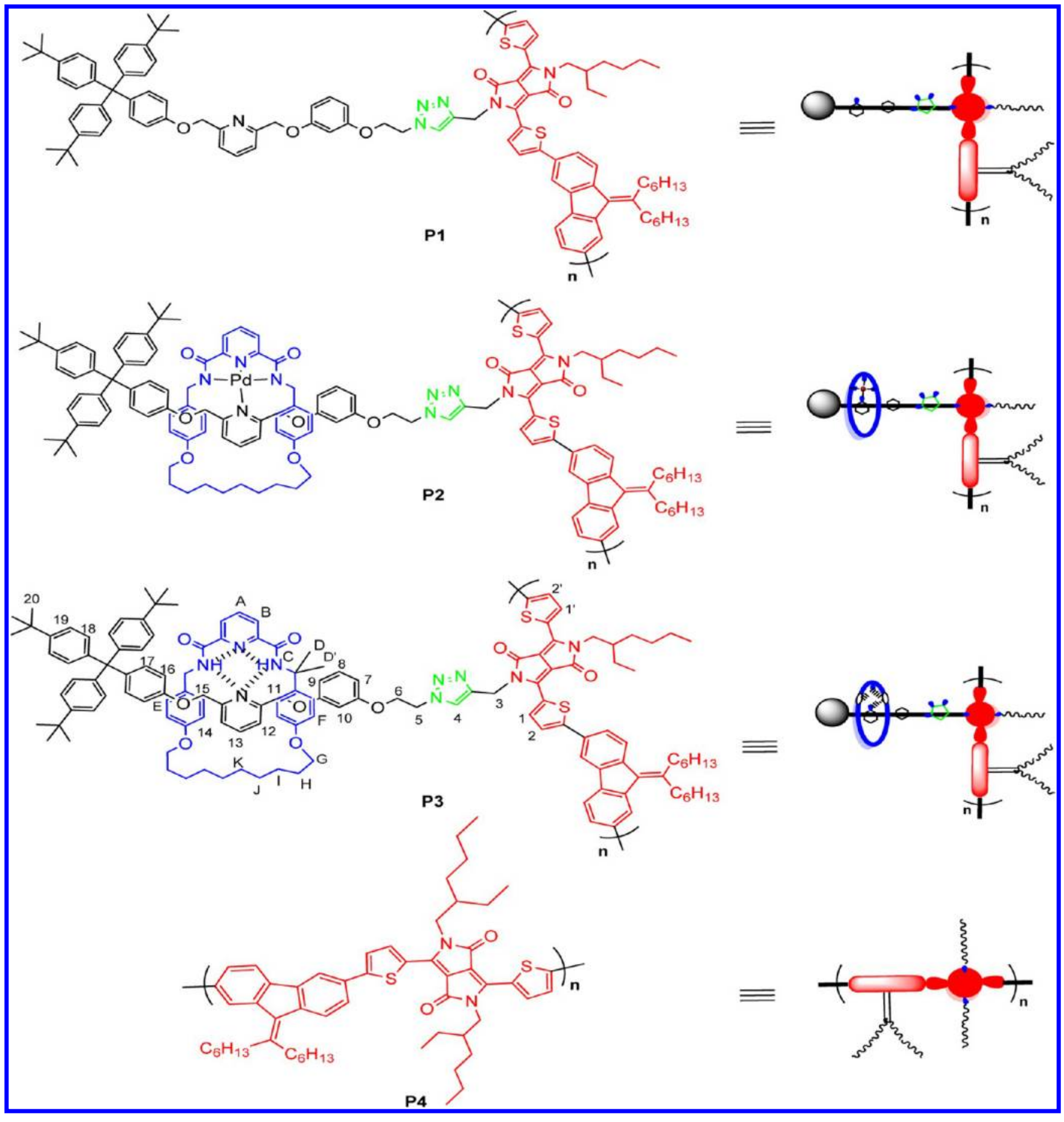

repeating polymer units, starting from smaller model units because a full large unit calculation is too extensive and lengthy. The calculations, including the simple asymmetric propargyl unit substituted diketopyrrolo pyrrole (D1), A1, and 9-alkylidene-9H-fluorene connecting to an asymmetric triazole-substituted diketopyrrolopyrrole unit (D2), were explored by quantum chemical calculations using the Gaussian09 software package. ${ }^{19}$ Geometry optimization of the ground state structures was carried out with DFT at the tHCTHhyb ${ }^{20}$ level of theory using the $6-31+\mathrm{G}(2 \mathrm{~d}, \mathrm{p})$ basis set. The excitation energies of the low-lying excited states and oscillator strengths were predicted using the time-dependent density functional theory (TD-DFT) with Handy's gradient-corrected correlation functionals at the HCTH147/6-31+G(2d,p) level. ${ }^{21}$ The orthogonal H-bonded strengths with and without the acid of demetalated [2] rotaxane monomer 2-b were examined using the semiempirical $P M 3^{22}$ method to understand the controlled nanostructure formation of the $\mathbf{P} 3$.

\section{RESULTS AND DISCUSSION}

Mechanically Interlocked Polymeric Architecture Design. It is expected that the introduction of unique topological cavities formed in situ by the virtue of mechanical bond into pendants of the polymeric architectures could be an ideal choice to realize the superior material processability. ${ }^{1 b, 6 d}$ Several merits, including the unique optical properties, flexible self-assembled nature of diketopyrrolopyrrole, ${ }^{23}$ and better coplanar structured 9-alkylidiene-9H-fluorene donor unit, ${ }^{24}$ 


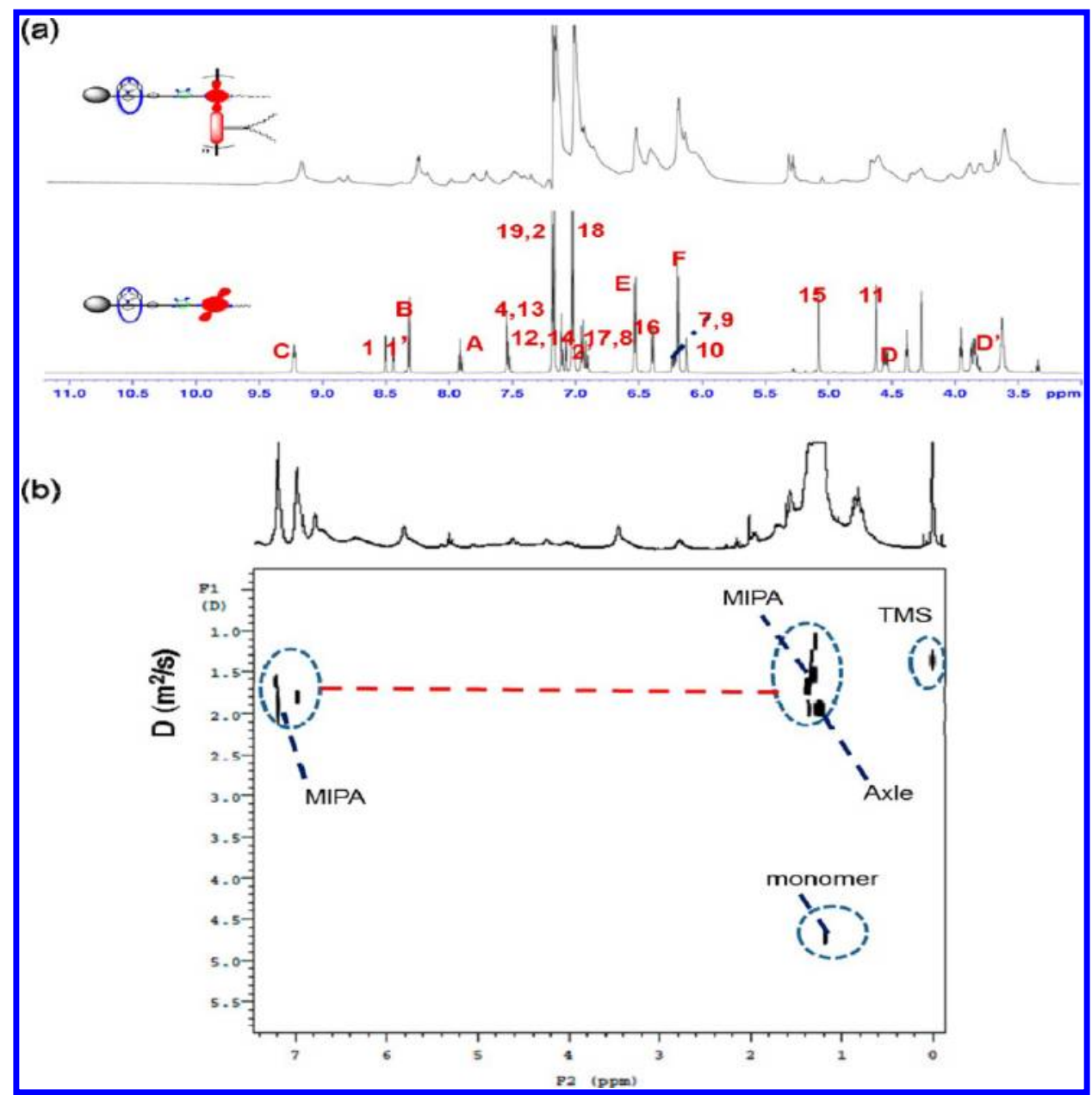

Figure 1. (a) ${ }^{1} \mathrm{H}$ NMR $\left(600 \mathrm{MHz}, \mathrm{CDCl}_{3}, 298 \mathrm{~K}\right)$ stock plot of monomer 2-b and polymer P3 and (b) 2D-DOSY (600 $\left.\mathrm{MHz}^{\mathrm{CDCl}} 3,298 \mathrm{~K}\right) \mathrm{NMR}$ spectra of P3 at a concentration of $12 \mathrm{mg} / \mathrm{mL}$. Diffusion coefficient of MIPA, axle part, and free monomer were marked with circles. The assignments correspond to the lettering shown in Scheme 1.

inspired us to accomplish the aforementioned task. The complete chemical structures of these polymers were depicted in Scheme 2, and the monomers 1-c, 2-a, and 2-b were prepared according to our previous protocol. ${ }^{18}$ The synthetic procedures and characterization data of all these polymers are provided in the Supporting Information.

Gel Permeation Chromatography (GPC) and 2D-DOSY NMR Characterization of MIPA. The molecular weights, polydispersity indexes (PDI), and self-assembled natures of these MIPAs were examined by GPC. In contrast to the polymer retention volume $(19.9 \mathrm{~mL})$ of simple $\mathbf{P 4}$, supramolecular polymer P3 showed a higher retention volume (22.1 $\mathrm{mL}$ ) as shown in Figure S1. Moreover, P3 revealed a narrower PDI value of 1.24 than P4 with a PDI value of 2.10. In contrast to $\mathbf{P 4}$, the interesting escalation of the retention volume in P3 with a higher molar mass [2] rotaxane unit was observed. It clearly suggested that a smaller molecular weight of P3 was obtained in contrast to P4, which was due to a lower degree of polymerization of P3 induced by its larger pendent group. Additionally, a larger retention volume could also be evolved in P3 via the more folded conformation during its macromolecular self-assembly owing to the strong intermolecular $\mathrm{H}$ bonding and $\pi-\pi$ stacking interactions as also suggested by Stoddart et al. ${ }^{25}$ However, the broad PDI of simple polymer P4 indicated a linear fashioned assembly through the $\pi-\pi$ stacking interactions.
To study the supramolecular polymerization, we compared the ${ }^{1} \mathrm{H}$ NMR spectra of both monomer 2-b and polyrotaxane P3 as shown in Figure 1a, in which broad peaks of P3 than those of 2-b clearly suggested that mechanically interlocked nature of P3 was maintained during polymerization. To confirm further the interlocked nature, we did protic demetalation of $\mathbf{P 2}$ in $\mathrm{CHCl}_{3} / \mathrm{MeOH}$ with potassium cyanide which gave P3 identical to that of obtained directly from monomer 2-b. Furthermore, 2D-DOSY experiments were probed to confirm the mechanically interlocked nature of $\mathbf{P 3}$ as depicted in Figure 1b, where the marked diffusion rate of P3 $\left(1.8 \times 10^{-10} \mathrm{~m}^{2} / \mathrm{s}\right)$ was clearly distinctive from the diffusion rate of free monomer $2-b\left(4.7 \times 10^{-10} \mathrm{~m}^{2} / \mathrm{s}\right)$. To further fortify the preserved mechanical interlocking nature of polymer P3, we conducted a 2D-DOSY experiment on the metalated polymer (P2) with the same degree of polymerization of P3. As shown in Figure S2, polymer $\mathbf{P 2}$ demonstrated a higher diffusion rate of $3.1 \times 10^{-10} \mathrm{~m}^{2} / \mathrm{s}$ in contrast to polymer P3 $\left(1.8 \times 10^{-10} \mathrm{~m}^{2} /\right.$ s). The slow diffusion rate ${ }^{14 a}$ of polymer P3 in comparison with the analogous monomer unit (2-b) as well as the metalated polymer (P2) lucidly supported the mechanically interlocked nature of the polymer through the strong orthogonal $\mathrm{H}$-bonded interactions. This result clearly denoted the well-preserved mechanical interlocking of P3 with globule-like assembly structures. 


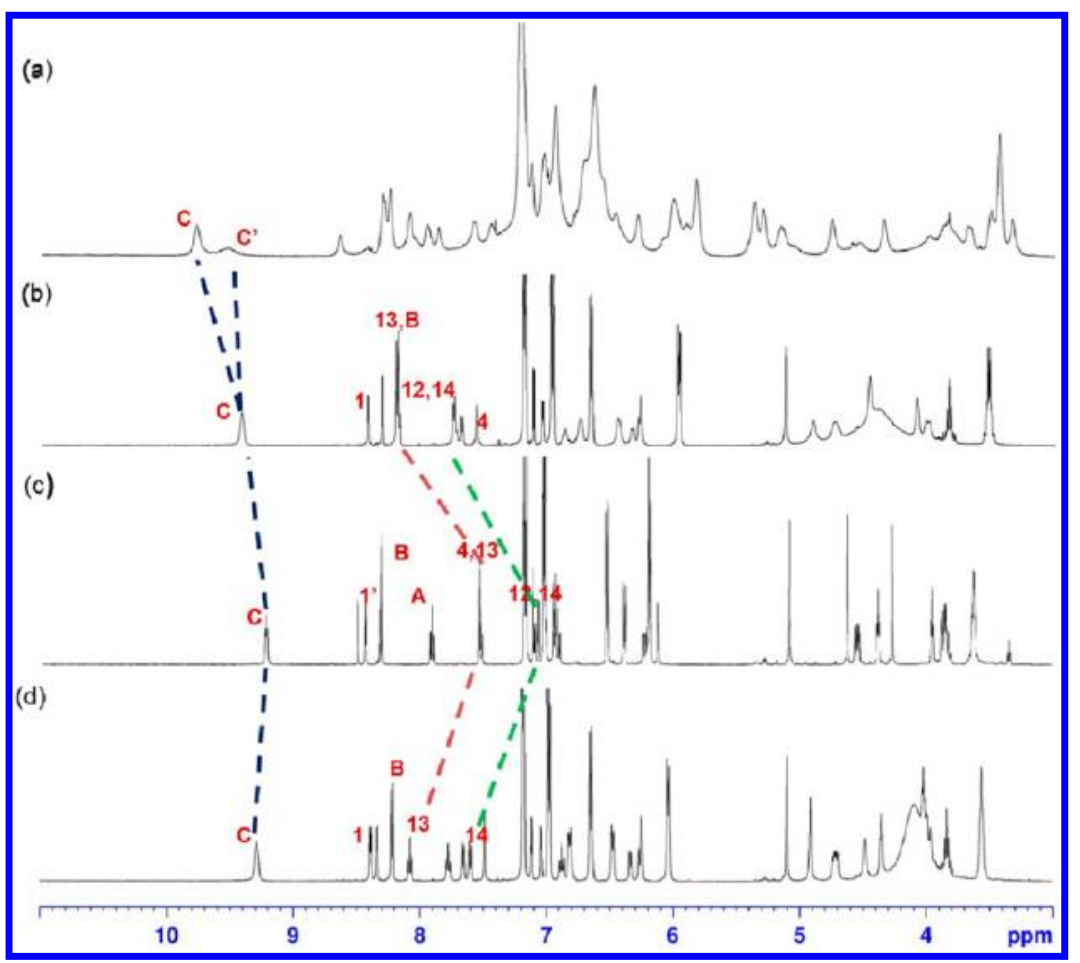

Figure 2. VT-NMR (500 MHz, $\mathrm{CDCl}_{3}$ ) stock plot of monomer 2-b in the presence of TFA: (a) 2-b + TFA (1.0 equiv), $233 \mathrm{~K}$; (b) 2-b + TFA (1.0 equiv), $298 \mathrm{~K}$; (c) free 2-b, $298 \mathrm{~K}$; (d) 2-b + TFA (1.0 equiv), $323 \mathrm{~K}$. The assignments correspond to the lettering shown in Scheme 1.

${ }^{1} \mathrm{H}$ NMR Spectroscopic Studies. To explore the stimulibased host-guest properties of these polymers, we carried out a series of ${ }^{1} \mathrm{H}$ NMR studies on the respective monomers of polymers. Upon adding TFA to monomer $\mathbf{2}-\mathbf{b}$, the wheel amide $\mathrm{NH}$ protons showed a downfield shift along with thread pyridyl protons $(12,13$, and 14) as shown in Figure S3. However, a downfield shift for proton $\mathrm{E}$ was observed during the titration of 2-b with TFA. Upon further addition no significant effects were evidenced, which clearly illustrated that TFA was deprotonated by the axle unit to induce a strong $\mathrm{H}$-bonded complex with the resulting trifluorocarboxylate anion. To further confirm the binding interactions, we verified the ${ }^{1} \mathrm{H}$ NMR spectrum of the dumbbell monomer 1-c with TFA; the noticeable downfield shifts for pyridyl protons 12,13 , and 14 supported the deprotonation of TFA by the pyridine station. Unambiguously, the Job plot analysis of 2-b by drawing the chemical shift changes against the mole fractions of TFA yielded a 1:1 stoichiometry as represented in Figure S4. Importantly, the acid-mediated host-guest interactions were completely reversible upon adding the base DBU as depicted in Figure S5, which proved the potential molecular switch ability of the mechanically interlocked polymeric architecture in polyrotaxane P3.

To further shed light on the acid-mediated dynamic selfassembly of MIPAs, variable temperature NMR (VT NMR) experiments of complex $\mathbf{2}$ - $\boldsymbol{b}$-TFA were conducted. When the temperature cooled down to $233 \mathrm{~K}$, the ${ }^{1} \mathrm{H}$ NMR spectrum of 2-b-TFA showed the splitted resonances for amide protons $\mathrm{C}$ and pyridyl protons 12,13 , and 14 along with aliphatic protons with broad peaks in contrast to that at $298 \mathrm{~K}$ as shown in Figure 2. However, when the solution of 2-b-TFA was heated to $323 \mathrm{~K}$ close to $d$-chloroform boiling point, interestingly the amide protons and pyridyl protons showed marginal upfield shifts. Moreover, 2- $\boldsymbol{b}$-TFA presented downfield shifts for amide and pyridyl protons along with aliphatic splitting at $323 \mathrm{~K}$ in comparison with free monomer 2-b at $298 \mathrm{~K}$. Prominently, 2-bTFA showed coalescence for the complex proton resonances at $298 \mathrm{~K}$ and represented that the self-assembly process of MIPAs could be evolved through folding and unfolding of individual rotaxane units. These observations plug to a positive cooperative effect of the thermally controllable folded conformation of the MIPA system. Furthermore, the kinetically stable self-assembly process through a continuous $\pi-\pi$ stacking in MIPAs was attributed to the steric demand of the polyrotaxane system with the interconvertible intermolecular orthogonal $\mathrm{H}$-bonded interactions and strong anion $-\pi$ and hydrophobic interactions along with high-energy unfolding of more individual MIPA units.

Orthogonal H-bonded interactions were further probed by the IR spectroscopy for rotaxane monomer 2-b and complex 2$\boldsymbol{b}$-TFA, where the vivid and strong H-bonded interactions could be visualized from Figure S6 with amide band at 3346 $\mathrm{cm}^{-1}$. The IR spectrum of 2 - $\boldsymbol{b}$-TFA presented a newly originated medium band at $2620 \mathrm{~cm}^{-1}$ with an amide strong band at $3331 \mathrm{~cm}^{-1}$. Moreover, the band at $1915 \mathrm{~cm}^{-1}$ corresponding to pyridine carboxylic acid type H-bonding band was shifted marginally to $1924 \mathrm{~cm}^{-1}$. The shift in the amide band along with a new band lucidly suggested a strong $\mathrm{H}$-bonded interaction between 2-b and TFA. We reasoned that the new band at $2620 \mathrm{~cm}^{-1}$ was instigated owing to the $\mathrm{H}$ bonded interactions of pyridinium trifluorocarboxylate anion.

Optical and Electrochemical Measurements. The photophysical properties of MIPAs were ideally measured to realize future optoelectronic devices and the absorption spectra in the UV-vis to NIR regions of these polymers in solution and solid films are shown Figures $\mathrm{S7a}$ and S7b, respectively. Interestingly, the polymers with rotaxane and dumbbell units (i.e., P3, P2, and P1) presented two bands, which illustrated one peak in the UV-vis region and another one in the NIR region in contrast to a single band in simple P4. Polymers P3 


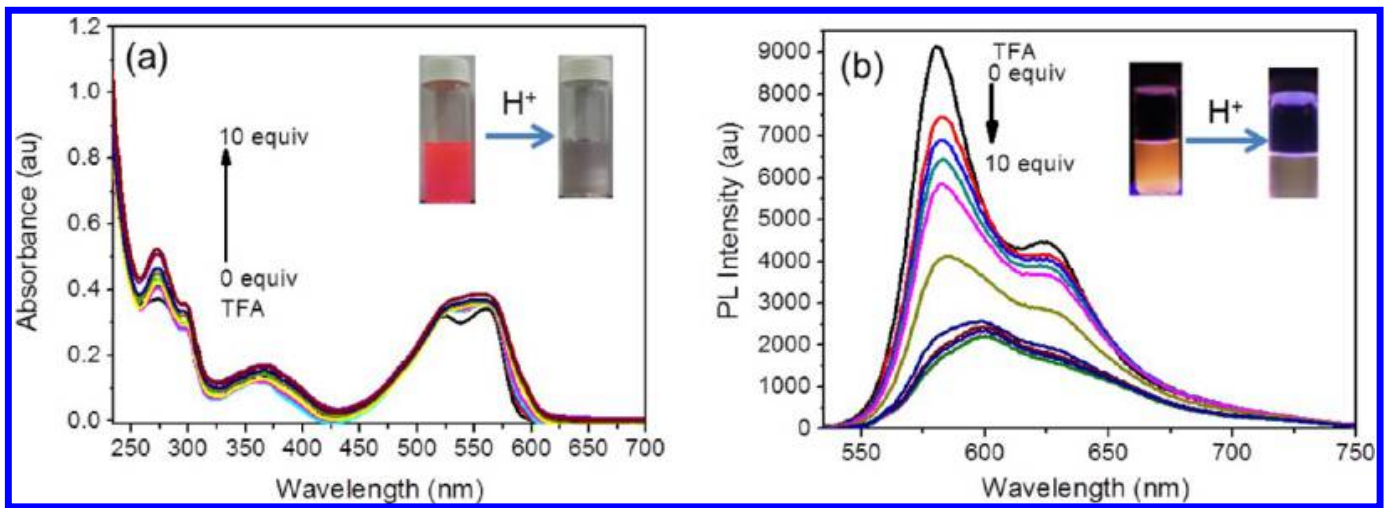

Figure 3. (a, b) UV-vis and fluorescence spectral changes of 2-b (10 $\mu \mathrm{M}$ in DCM) upon the addition of TFA in DCM (0-10 equiv), respectively. Insets: pictures in (a) and (b) indicate naked eye color changes as well as fluorescence changes under UV light (365 nm) upon the addition of TFA, respectively $\left(\lambda_{\mathrm{ex}}=525 \mathrm{~nm}\right)$.

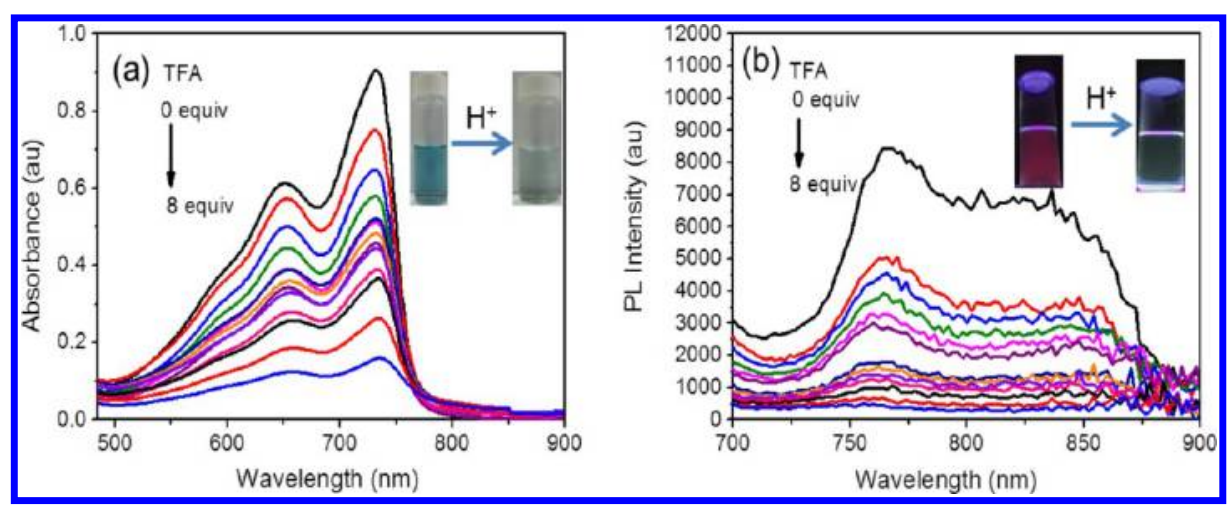

Figure 4. (a, b) UV-vis and fluorescence spectral changes of $\mathbf{P 3}$ (10 $\mu \mathrm{M}$ in THF) upon the addition of TFA in THF (0-8 equiv), respectively. Insets: pictures in (a) and (b) indicate naked eye color changes as well as fluorescence changes under UV light (365 nm) upon the addition of TFA, respectively $\left(\lambda_{\mathrm{ex}}=720 \mathrm{~nm}\right)$.

and P4 in solution and solid films showed the absorption maximum values at 642,725 (solution)/664, 746 (solid) nm and 622 (solution)/643 (solid) nm. An obvious red-shift (20 $\mathrm{nm}$ ) from solution to films for P3 and P4 was observed; however, both shifts from solution to films in $\mathbf{P 1}$ and $\mathbf{P 2}$ were trivial. This typical behavior of polymers in the film case could be ascribed to a proliferation in the effective conjugation length due to a better coplanar geometry of both polymer backbones in the solid state. On the other hand, in contrast to simple P4, the prominent absorption band in the NIR region for rotaxanated polymers demonstrated that the pendant dumbbell and [2] rotaxane units enabled effective molecular packing and intramolecular charge transfer interactions and thus to led to extended conjugations in these MIPAs.

Encouraged by the excellent photophysical properties of rotaxanated conjugated polymers, we decided to explore acidmediated host-guest complexation by UV-vis and PL titrations as shown in Figure 3a. The two absorption maxima values at 525 and $554 \mathrm{~nm}$ of $2-\mathrm{b}(10 \mu \mathrm{M})$ became a single broad absorption with an incremental trend; simultaneously, the emission intensity at $580 \mathrm{~nm}$ of $\mathbf{2}$-b was gradually decreased (Figure $3 b)$ during the titration with TFA ( $0-10$ equiv) in dichloromethane. Additionally, it can be confirmed by a perceptible naked eye and fluorescence color changes as illustrated in the insets of Figures $3 \mathrm{a}$ and $3 \mathrm{~b}$.

These observations were attributed to a deprotonation of TFA by 2-b (pyridine site) and in situ formation of a strong $\mathrm{H}$ bonded complex between the resulting trifluorocarboxylate anion, 2,6-bis(carboxyamido)pyridine, and the pyridinium part of the dumbbell. Unlike the monomer case, polyrotaxane P3 $(10 \mu \mathrm{M})$ provided exclusively different absorption patterns during the titration with TFA ( $0-8$ equiv) in THF; the absorption bands of polyrotaxane P3 at 642 and $725 \mathrm{~nm}$ gradually decreased in their intensities as displayed in Figure 4a. Similarly, the fluorescence emission maxima at $766 \mathrm{~nm}$ decreased concurrently as shown in Figure $4 \mathrm{~b}$. During the titration with TFA, the absorption peak at $642 \mathrm{~nm}$ was completely vanished while 8 equiv of TFA was added in P3. As a result, a noticeable naked eye color change was observed as presented in the inset of Figure 4a.

The binding constants of $\mathbf{2}-\mathbf{b}$ and P3 with TFA were calculated to be ca. $9.9 \times 10^{3}$ and $1.22 \times 10^{5} \mathrm{M}^{-1}$, respectively, as shown in Figures S8a and S8b. Compared with monomer 2b, the drastically improved binding constant of polyrotaxane P3 clearly indicated an enhanced interaction of acid with polymer has more binding sites to augment the supramolecular electronic energy transfer between the polymer backbone and biding cavity. ${ }^{6 \mathrm{~d}, 9 \mathrm{c}}$ Moreover, to fortify the selectivity of the orthogonal H-bonded cavity in $\mathbf{2}-\mathbf{b}$ and $\mathbf{P} \mathbf{3}$, control experiments were conducted on other monomers and polymers. However, their responses were trivial under similar conditions (see Figure S9a-d).

Delightfully, the complexation of TFA with the orthogonal H-bonded unit in 2-b and P3 was completely reversible upon adding the excess of base DBU. The on-off-on fluorescence etiquette for both 2-b and P3 was successfully achieved via an 


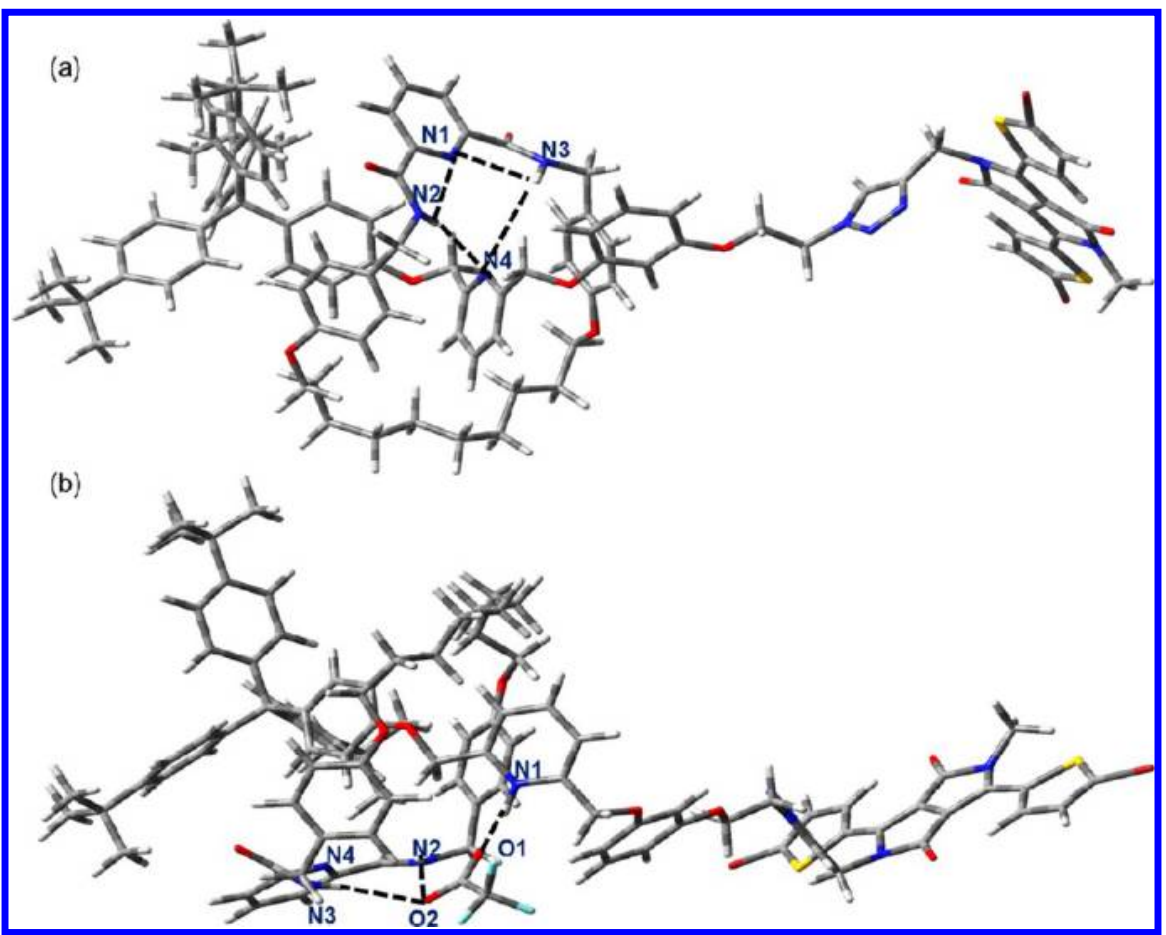

Figure 5. (a, b) PM3 optimized chemical structures of monomer 2-b and complex 2-b-TFA, respectively. Selected H-bonded lengths [ $\AA$ ] for 2-b:

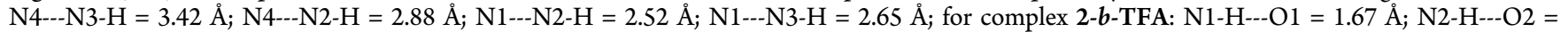
$2.56 \AA ̊$; N3-H---O2 = $2.77 \AA ̊$.

Table 1. TD-DFT Calculated HOMO, LUMO, HOMO-LUMO Gap (HLG), Wavelength $\left(\lambda_{\max }\right)$, and Oscillator Strength $(f)$ for D1, D2, and A1

\begin{tabular}{|c|c|c|c|c|c|c|c|c|}
\hline \multirow[b]{2}{*}{ compd } & \multirow[b]{2}{*}{ HOMO $(\mathrm{eV})$} & \multirow[b]{2}{*}{ LUMO (eV) } & \multirow[b]{2}{*}{$\mathrm{HLG}^{a}(\mathrm{eV})$} & \multicolumn{5}{|c|}{ TD-DFT calculated transition data ${ }^{b}$} \\
\hline & & & & excited state & $\lambda_{\text {abs,cal }}^{b}(\mathrm{~nm})$ & $\overline{f^{c}}$ & config $^{d}$ & weight $^{e}(\%)$ \\
\hline \multirow[t]{3}{*}{ D1 } & -5.19 & -3.01 & 2.18 & S1 & 566 & 0.431 & $\mathrm{H} \rightarrow \mathrm{L}$ & 96 \\
\hline & & & & S2 & 433 & 0.001 & $\mathrm{H}-1 \rightarrow \mathrm{L}$ & 38 \\
\hline & & & & & & & $\mathrm{H} \rightarrow \mathrm{L}+1$ & 48 \\
\hline \multirow[t]{5}{*}{ D2 } & -4.90 & -2.90 & 2.00 & S1 & 666 & 0.790 & $\mathrm{H} \rightarrow \mathrm{L}$ & 72 \\
\hline & & & & & & & $\mathrm{H} \rightarrow \mathrm{L}+1$ & 27 \\
\hline & & & & S2 & 602 & 0.373 & $\mathrm{H} \rightarrow \mathrm{L}+1$ & 60 \\
\hline & & & & & & & $\mathrm{H} \rightarrow \mathrm{L}$ & 22 \\
\hline & & & & & & & $\mathrm{H}-1 \rightarrow \mathrm{L}$ & 16 \\
\hline \multirow[t]{3}{*}{ A1 } & -5.53 & -1.75 & 3.78 & S1 & 409 & 0.016 & $\mathrm{H} \rightarrow \mathrm{L}$ & 97 \\
\hline & & & & S2 & 330 & 0.142 & $\mathrm{H}-1 \rightarrow \mathrm{L}$ & 53 \\
\hline & & & & & & & $\mathrm{H}-1 \rightarrow \mathrm{L}+1$ & 36 \\
\hline
\end{tabular}

${ }^{a}$ HOMO, LUMO, and HOMO-LUMO gaps are calculated with the tHCTHhyb/6-31+G(2d,p) method. ${ }^{b}$ Absorption energies are calculated with the TDDFT method at the HCTH147/6-31+G(2d,p) level. ${ }^{c}$ Oscillator strength. ${ }^{d} \mathrm{H}$ and L stand for the predicted HOMO and LUMO, respectively. The second highest occupied and lowest unoccupied molecular orbitals denoted as $\mathrm{H}-1$ and $\mathrm{L}+1$, respectively. ${ }^{e}$ Only configurations with $5 \%$ or greater contribution are included.

alternate addition of acid and base up to four cycles as shown in Figure S8c-f. Hence, the absorption patterns in polyrotaxane P3 can be assigned to more mobile $\mathrm{H}$-bonded interactions between interlayered polymer axle pyridinium units and trifluorocarboxylate anion along with anion $-\pi$ interactions, thus the pendant rotaxane imparting unique photophysical properties to the $\pi$-conjugated backbones of MIPAs.

The electrochemical properties of monomers and polymers were evaluated by cyclic voltammetry (CV) measurements. Both polyrotaxane $\mathbf{P 3}$ and monomer 2-b exhibited the quasireversible redox process as shown in Figures S10a and S10b. The energy levels of highest occupied molecular orbital (HOMO) and lowest unoccupied molecular orbital (LUMO) of P3 were compared with those of its precursor monomer 2-b.
It was found that $\mathbf{P} 3$ possessed a higher HOMO level $(-5.19$ $\mathrm{eV})$ than 2-b $(-5.35 \mathrm{eV})$ and a lower LUMO level $(-3.45 \mathrm{eV})$ than 2-b $(-3.17 \mathrm{eV})$, which suggested a narrower bandgap acquired in polyrotaxane P3. These energy level changes for P3 could be ascribed to the extended conjugation length in the polymer with the addition of the electron-rich AKF unit. Detailed optical and electrochemical data are summarized in Table S1.

Theoretical Calculations. To corroborate the experimental observation of the polymers and to provide a better insight into fundamental orthogonal $\mathrm{H}$-bonded cavity and TFA binding interaction with a subtle change of macromolecular architecture, theoretical calculations were carried out as described above. For the sake of dealing with the H-bonded 
interactions in polyrotaxane $\mathbf{P}$ 3, its precursor monomer $\mathbf{2}-\mathbf{b}$ was used in this study by replacing the 2-ethylhexyl unit with a methyl group. PM3 optimized energy minimized geometry of 2-b ([2] rotaxane monomer) is given in Figure 5a, illustrating that the macrocycle in free $\mathbf{2}-\mathbf{b}$ monomer was tilted with an angle of $26^{\circ}$ to avoid intercomponent pyridyl lone pair interactions with an unsymmetrical intercomponent hydrogen-bonded distances of 2.8 and $3.2 \AA$ in a twisted chair conformation. However, when TFA was added to $\mathbf{2}-\mathbf{b}$, the cycle displaced from central pyridine station relative to the free $\mathbf{2}-\mathbf{b}$ as shown in Figure $5 \mathrm{~b}$ with a tilt angle of $45^{\circ}$.

A further Mulliken charge analysis clearly revealed that the axle pyridine deprotonated the TFA (axle pyridinium $\mathrm{NH}^{+}$ $\mathrm{N}(1)=0.43 e, H=0.2 e, \mathrm{O}(2)=-0.45 e$, and $\mathrm{O}(1)=-0.64 e$, where $e$ is the magnitude of the charge on an electron), forming a strong intercomponent hydrogen-bonded complex 2-b-TFA with the resulting trifluorocarboxylate with distances of N1-H--$\mathrm{O} 1=1.67 \AA$, N2-H---O2 = $2.56 \AA$, and N3-H---O2 = $2.77 \AA$, respectively. The evolution of molecular orbital delocalization for the HOMO and LUMO orbital density states in 2-b showed that in both these density states electron delocalization is mainly residing on the diketopyrrolopyrrole units in the free 2$\mathbf{b}$ as shown in Figure S11a,b. We envisioned that the addition of TFA might change the electronic distribution such that the HOMO corresponded to the diketopyrrolopyrrole part and the LUMO corresponded to the TFA-2-b interaction site. Indeed, we found this to be the case as shown in Figure S11c,d. These two prevailing theoretical observations were consistent with our above ${ }^{1} \mathrm{H}$ NMR and optical measurements as well as the previous report. ${ }^{26}$

To provide the further clue about the origin of extended conjugations in these polymers, we employed the DFT and TD-DFT calculations. The predicted absorption wavelengths $\left(\lambda_{\text {abs}}\right.$, cal $)$, oscillator strengths $(f)$, and the configuration description of the lowest two singlet excited states are shown in Table 1. To establish the role of individual units on the effective $\pi$-conjugations in these polymers, we optimized the simple A1 and D1 units first; the energy-minimized planar structures and the electronic delocalizations of the frontier molecular orbitals (FMOs) for A1 and D1 can be visualized in Figures S12 and S13, respectively.

For both A1 and D1 units, their lowest energy singlet transitions are mainly from HOMO to LUMO. However, in the next excited state, we noticed a major highest energy singlet transition in $\mathbf{D l}$ from HOMO to LUMO+1 and in the $\mathbf{A l}$ from HOMO-1 to LUMO as well as HOMO-1 to LUMO+1. For both units FMOs were delocalized over the entire molecule. To substantiate the above observations and the trend, we carried out the TD-DFT calculations. We noticed a remarkable incremental shift in the oscillator strength $(f)$ of A1 from the ground state to the next excited state (0.016 to 0.142$)$, whereas the $f$ of $\mathbf{D} 1$ was noted to decrease from 0.431 to 0.001 as shown in Table 1 .

These observations suggested that both molecules possessed a strong $\pi$-character and gave a clue that the assembly of these two units could enable better intramolecular charge transfer transitions. Taking this vivid theoretical clue, we further connected the triazole-substituted asymmetric diketopyrrolopyrrole and A1 units to afford the D2 unit (via the Suzuki coupling strategy) as well as to model the ground and excited states to investigate the consequential electronic properties of these polymers.
The optimized coplanar conformation of D2 and FMO delocalizations of HOMO and LUMO are presented in Figure 6. For the D2, the two units were almost fully coplanar while

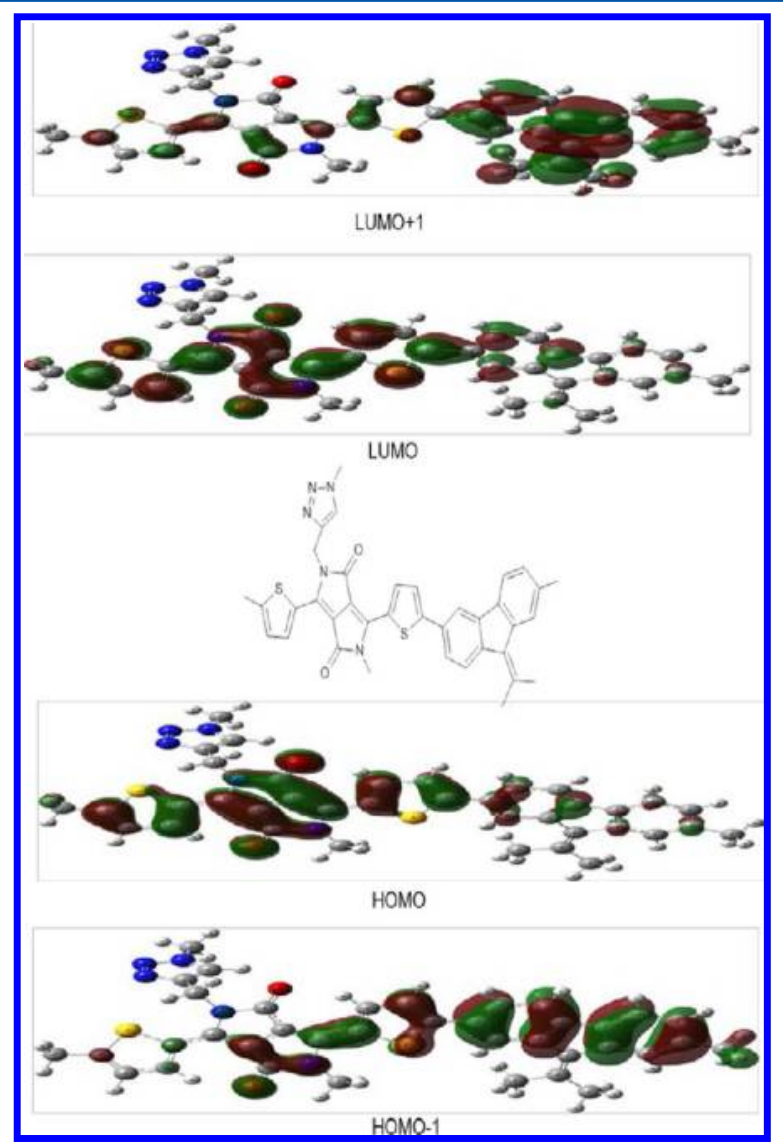

Figure 6. TD-DFT optimized stable chemical structures of monomer D2 modeled for the polymer in this study. Molecular orbital distribution plots of HOMO and LUMO states in the ground state and their corresponding $\mathrm{HOMO}-1$ and $\mathrm{LUMO}+1$ plots in the excited state.

the pendant substituted triazole unit demonstrated a model of the dumbbell units presenting a twist angle of $56^{\circ}$ against the D1 unit as shown in Figure S14. Moreover, the inserted triazole substitution was coplanar with the D1 unit, the main chain became distorted between the D1 and A1 units with a twist angle of $24^{\circ}$. The analysis of FMO energy levels revealed that the major energy transition in the ground state of $\mathbf{D} 2$ was mainly from HOMO to LUMO; however, in the next excited state the major energy transition contribution resulted from $\mathrm{HOMO}$ to $\mathrm{LUMO}+1$. Because of the enhanced main-chain conjugation length by the connection of the D1 and A1 units in D2, the HOMO energy level raised to ca. $0.29 \mathrm{eV}$ while the LUMO dropped by $0.11 \mathrm{eV}$.

These results strongly indicated that the enriched intramolecular charge transfer transitions facilitated the marginal destabilization of the HOMO and the stabilization of the LUMO, thus narrowing the band gap for D2 with red-shifts in all of these polymers. Moreover, the NIR absorption band in the case of polyrotaxane P3 may be ascribed to an effective molecular packing and unperturbed backbone $\pi$-conjugation owing to its controlled topological bonding. On the basis of these theoretical results, we can infer that the introduction of a topological bond in the $\pi$-conjugated polymers may expedite 


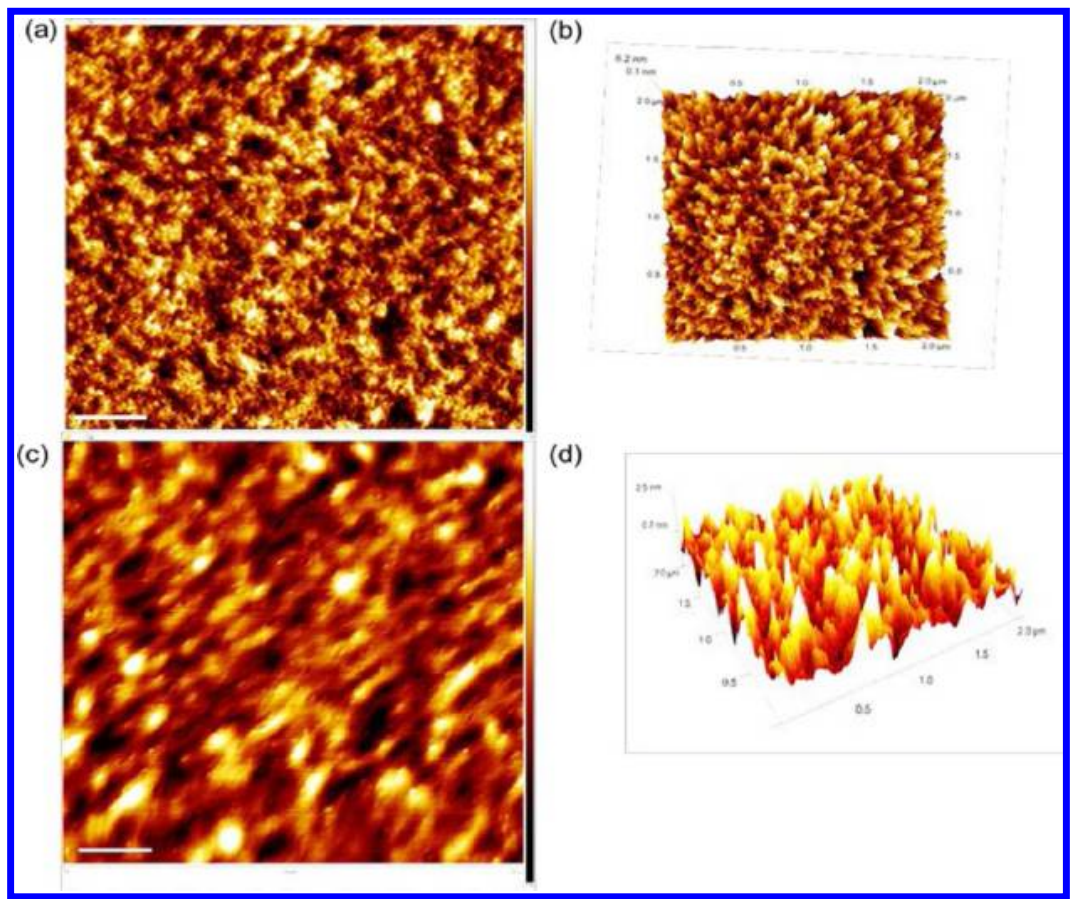

Figure 7. (a, c) AFM images of polymers P2 and P3 (100 $\mu$ M solutions in THF), respectively. (b, d) Rendered AFM-3D images of (a) and (b), respectively. Scale bar (a, b) $=2 \mu \mathrm{m} ; z$-scale $(\mathrm{a})=6.2 \mathrm{~nm}$ and $(\mathrm{c})=2.5 \mathrm{~nm}$.

the effective backbone conjugation by the enhanced molecular packing which was mainly governed by the orthogonal $\mathrm{H}$ bonding and the $\pi-\pi$ stacking interactions offered by the controlled topological cavities.

Thermal properties of these polymers were probed by thermogravimetric analysis (TGA). The 5\% weight loss temperatures $\left(T_{\mathrm{d} 5}\right)$ of polymers P4 and P3 were 320 and $344{ }^{\circ} \mathrm{C}$, respectively, as shown in Figure S15. The high thermal stability of polyrotaxane $\mathbf{P} \mathbf{3}$ was attributed to its folded nature presumably caused by flexible topological cavities of pendant rotaxane unit in $\mathbf{P 3}$.

To understand the effect of the orthogonal H-bonded rotaxane on macromolecular architecture packings and crystalline properties, powdered X-ray diffraction patterns of these polymers were evaluated. The polymer P4 X-ray diffractogram showed a characteristic reflection at $2 \theta=6.27$ $\AA(d$-spacing $=14.08 \AA)$ referenced to the interlayered distance between polymer chains and $2 \theta=24.81 \AA(d=3.58 \AA)$ which denoted the $\pi-\pi$ stacking interactions of the polymer units (see Figure S16). However, in the case of polyrotaxane P3, these reflections were shifted to $2 \theta=9.46 \AA$ ( $d$-spacing $=9.33$ $\AA$ ) and $2 \theta=18.41 \AA$ ( $d$-spacing $=4.81 \AA$ ), respectively. Moreover, in the case of P3, the degree of crystallinity was reduced and eventually enhanced the organic solubility of this polymer in relative to other polymers in this study. This gave a strong evidence that the introduction of a pendant orthogonal $\mathrm{H}$-bonded [2] rotaxane was regulating the $\pi$-conjugated backbone polymer molecular arrangement. This result is consistent with our above theoretical observations as well as the other previous reports. ${ }^{5 c, e}$

Microscopic and DLS Monitoring of Controllable Hierarchical Nano Self-Assemblies of MIPAs. Cyclodextrin- and cucurbituril-based molecular self-assemblies of polyrotaxanes were extensively researched. ${ }^{27}$ However, the studies based on pendant orthogonal H-bonded rotaxane copolymers are due owing to their complexities in attaining the well-organized molecular packing at the molecular level.

We have primarily conducted atomic force microscopy experiments to study the preserved mechanical interlocking nature of polymer P3 and the effect of the pendant rotaxane unit on the polymer backbone. The optimized dilute solutions $(100 \mu \mathrm{M})$ of polymers $\mathbf{P 2}$ and P3 in THF were used to avoid possible interchain aggregation behavior as shown in Figures $7 \mathrm{a}$ and $7 c$, respectively. We noticed a change in the morphology from the buddle-like structure with the root-mean-square roughness $(S q)$ of $1.7 \mathrm{~nm}$ in $\mathbf{P 2}$ to the globule-like structure with $S q=0.6 \mathrm{~nm}$ in P3. Moreover, the rendered 3D images of both P2 and P3 showed the smooth and well aligned surfaces in Figures $7 \mathrm{~b}$ and $7 \mathrm{~d}$, respectively. Cross-sectional analyses of these polymers further confirmed the morphological evolution from the metalated rotaxane polymer to demetalated rotaxane polymer as shown in Figure S17. Furthermore, we recorded the AFM images of the simple polymer P4 and MIPA (P3). As shown in Figure S18a, P4 illustrated a higher roughness $(S q=$ $16.1 \mathrm{~nm}$ with an average cross-sectional height of $35 \mathrm{~nm}$ ) than P3 $(S q=0.6 \mathrm{~nm}$ with an average cross-sectional height of 1.5 $\mathrm{nm})$.

As shown in Figure S19, the structural length and depth analyses of polymers showed a smaller average height $(4.2 \mathrm{~nm})$ in polymer $\mathbf{P 3}$ in contrast to $\mathbf{P 2}(8.3 \mathrm{~nm})$ and $\mathbf{P 4}(38 \mathrm{~nm})$. Thus, this result indicated that compared with $\mathbf{P 4}$, the pendant orthogonal H-bonded unit in polyrotaxane P3 has an ability not only to form interconvertible $\mathrm{H}$-bonding with the other units in the polymer but also to affect the self-assembly of the conjugated backbone. More prominently, these results indicated that the mechanical bond of $\mathbf{P} 3$ was well preserved during the demetalation and polymerization processes via $\mathrm{H}$ bonding, $\pi-\pi$ stackings, ${ }^{28}$ and hydrophobic interactions. Both the finite radius of the probe tip and loading force applied to the sample preserve a stable controlled feedback and eventually 


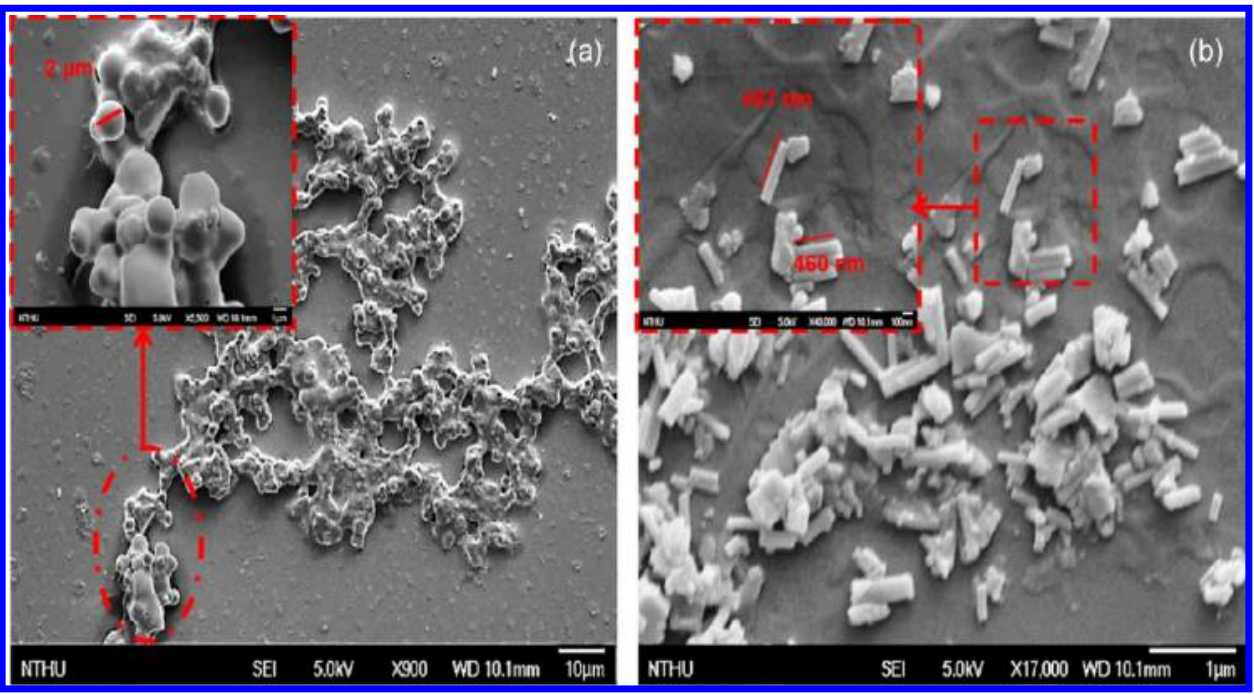

Figure 8. FE-SEM images of polymer P3 (a) in free state and (b) in protonated state with the addition of TFA (1 M, 10 equiv in THF). Insets: the corresponding enlarged portions of (a) and (b), respectively. Scale bar (a) $=10 \mu \mathrm{m}$ and (b) $=1 \mu \mathrm{m}$; insets: scale bar $(\mathrm{a})=1 \mu \mathrm{m}$ and (b) $=100 \mathrm{~nm}$.

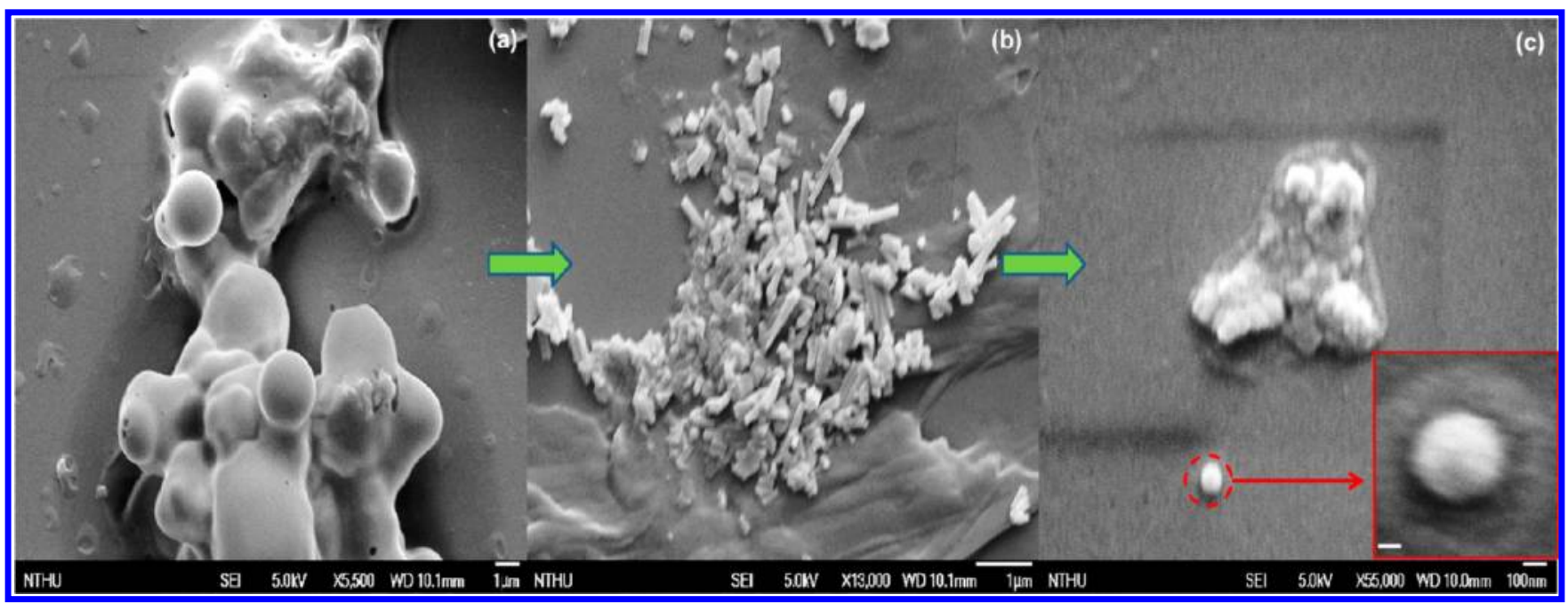

Figure 9. FE-SEM images of polymer P3 (100 $\mu$ M solution in THF): (a) in free state, (b) in the protonated state with the addition of TFA (1 M, 10 equiv), and (c) in the recovered state by neutralization with DBU. Inset in (c) magnified portion of spherical vesicle. Scale bar $(\mathrm{a}, \mathrm{b})=1 \mu \mathrm{m}$ and $(\mathrm{c})$ $=100 \mathrm{~nm}$; inset of $(\mathrm{c})=100 \mathrm{~nm}$.

control the structural and molecular dimensions of this MIPA. $^{29}$

We are intrigued in exploring the controlled self-assembly structures of MIPAs as well as the pivotal issue of the external stimuli (acid-base) effect in a reversible manner on the selfassembly of polymer P3. The SEM images revealed spherical vesicular type particles that appeared to be flattened and bloated due to the sample preparation as displayed in Figure 8a. However, the protonated form presented a distinctive flake as well as nanofiber structures, and the average size of the nanofibers is estimated to be $460 \mathrm{~nm}$ (Figure 8b). Moreover, the huge-sized (micrometers) spherical vesicles could be inevitable owing to its inherent folded globules geometry as well as the process of surface energy minimization ${ }^{1 \mathrm{~b}, 30}$ during the self-assembly process.

To substantiate such an expedient phenomenon further, we devoted to study the effect reversibility of these MIPAs. One could imagine that the protonation of the orthogonal $\mathrm{H}$ bonded unit in P3 would strongly influence the morphology as suggested by our above VT-NMR, photophysical, and theoretical studies. Delightfully, a drastic change in morphology was indeed observed after protonation of polyrotaxane P3 by TFA ( $1 \mathrm{M}, 10$ equiv) as shown in Figure 9b, which formed a flake-like structure as well as nanofiber structures in contrast to its spherical vesicles of free P3. Fairly, the reversible molecular switch ability was achieved by treating P3-TFA with DBU base as shown in Figure 9c, which is almost identical to its spherical vesicles shape as displayed in Figure 9a. The acid-base controllable morphological changes were reasonable as the protonation of the axle unit in P3 by TFA disrupted the selfassembly process due to the electrostatic repulsions. However, to minimize such repulsions, complex P3-TFA would reorient themselves in an elongated nucleation growth fashion to present hierarchical stacks with strong $\pi-\pi$ stacking interactions.

Superior to SEM, we recorded the TEM images to get rough insights into the sizes and shapes of these hierarchical nano selfassembled structures. The TEM image (Figure 10a) of polyrotaxane P3 showed spherical vesicular type particles comparable to SEM images as we noticed earlier, where the size 


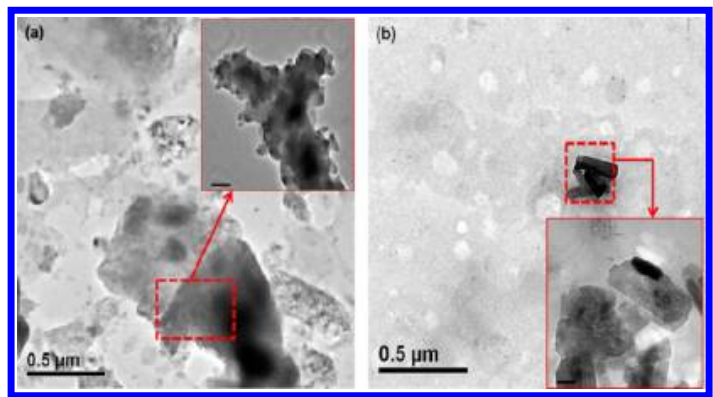

Figure 10. TEM images of polymer P3 (a) in free state and (b) in protonated state with the addition of TFA ( $1 \mathrm{M}, 10$ equiv in THF). Insets: the corresponding enlarged portions of (a) and (b), respectively. Scale bar: $(\mathrm{a}, \mathrm{b})=500 \mathrm{~nm}$; insets: scale bar $(\mathrm{a})=200$ $\mathrm{nm}$ and $(\mathrm{b})=100 \mathrm{~nm}$.

of vesicular type particle was estimated to be an average size of $120 \mathrm{~nm}$ from Figure S20a. Exclusively, the TEM image revealed the picture lucidly in the case of protonated P3 which presented the multilayered hexagonal-shaped nanosheets ${ }^{31}$ as shown in Figure 10b. We attempted to disclose the sizes and shapes of the individual sheets to understand the assembly mechanism. However, the TEM image depicted that the individual sheets looked to be embedded in to multilayered hierarchical hexagonal nanosuperstructures, where the average size of the nanosheets was estimated to be $260 \mathrm{~nm}$ (with a height of $120 \mathrm{~nm}$, see Figure S20b).

Both force and electron microscopy images indicated that such a pronounced size of these vesicular and hexagonal sheets seemed to well surface-adhered and flattened upon evaporation of solvents from their cavities. ${ }^{32}$ Furthermore, we carried out DLS measurements of $\mathbf{P 3}$ in THF solution which revealed an average hydrodynamic diameter of vesicles ca. $140 \mathrm{~nm}$ and hexagonal sheets ca. $440 \mathrm{~nm}$ in its free and protonated forms, respectively (Figure S21). These results are in excellent agreement with the above morphologies of SEM and TEM observations. More importantly, upon adding base to the protonated P3, it morphed back into the vesicle state (from sheet state) with an average diameter of $150 \mathrm{~nm}$, which showed a marginal difference in the average diameter owing to the precipitated salts during neutralization. Therefore, on the basis of this concrete evidence, we deduced that the pendant orthogonal H-bonded cavity in P3 adopted folded geometry by virtue of mechanical bond and prone to self-assemble into the bimolecular-layered structure in tail-to-tail fashion, where the rotaxane units were stacked exteriorly via the $\pi-\pi$ stacking and larger alkyl chains oriented interiorly owing to their hydrophobic interactions as shown in Figure 11. Moreover, upon adding TFA to P3, it tended to reorganize to minimize the local electrostatic repulsions between protonated axle parts as well as to stack further in layer-by-layer fashion to form hierarchical nanosheets and fibers with lateral $\pi-\pi$ stackings along with an inevitable cooperative interlayered anion $-\pi$ interactions. Eventually, the orthogonal H-bonds in P3 not only presented controllable hierarchical nanoself-assembly but also induced a better material processability relative to the other polymers (P1, P2, and P4).

\section{CONCLUSIONS}

In this study, we have developed a novel polymer with the mechanically interlocked polymeric architecture by polymerization of 9-alkylidene-9H-fluorene with a diketopyrrolopyrrole tethered with an orthogonally $\mathrm{H}$-bonded macrocycle unit, which successfully illustrated the effects of orthogonal $\mathrm{H}$ bonded unit on the photophysical properties as well as nano self-assembly process of the conjugated main-chain polymer (P3). It demonstrated spectroscopically and microscopically that the mechanically interlocked nature of polyrotaxane P3 was well preserved during the polymerization. Benefiting from its unique topological cavities formed in P3, it showed enriched material properties, such as extended conjugations, narrow bandgaps, and unique self-assembled structures, in contrast to the other polymers in this study. Remarkably, the fluorescence on-off-on reversible acid-base controllable molecular switch ability in NIR region for the first time has been fruitfully attained by supramolecular electronic energy transfer between the polymeric backbone and pendent topological cavities. We were able to show that the orthogonal $\mathrm{H}$-bonded unit in P3 induced the hierarchical nanostructures, first into vesicle-shaped particles in its free form, which further morphed into hexagonal nanosheets and fibers in its protonated form. The formation of vesicle-type nanostructures was mainly governed by $\pi-\pi$ stackings and hydrophobic interactions by virtue of its folded geometry. Moreover, this polymer in protonated state was reoriented to elude local electrostatic repulsions and augmented positive cooperative anion $-\pi$ interactions between embedded inner layers were also allowed to form hierarchical multilayered hexagonal nanosheets and fibers Therefore, we developed the first prototype design of a polyrotaxane architecture and set a stage to realize previously unattainable macromolecular architectures with orthogonally H-bonded topological cavities in a reversible fashion. Hence, this design of functional

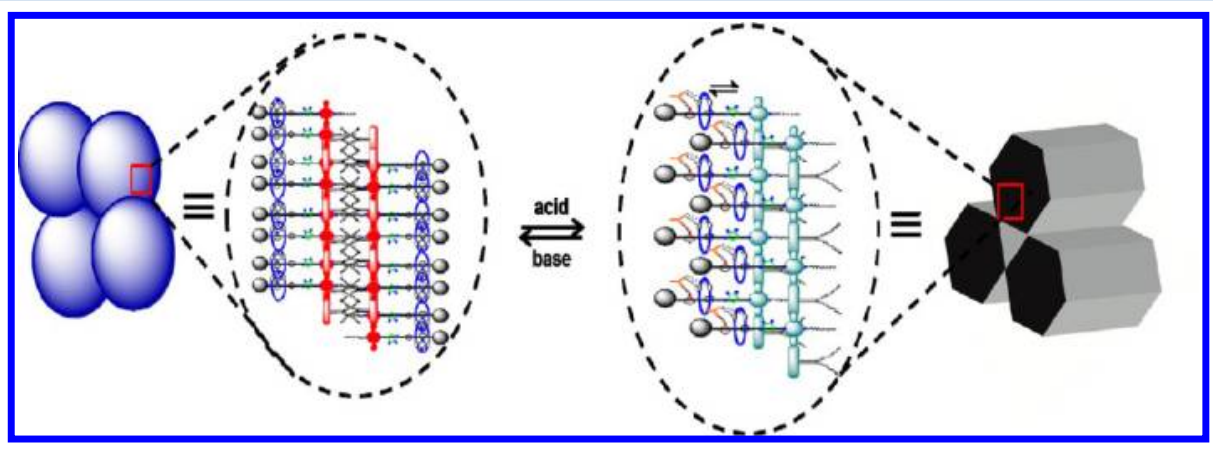

Figure 11. Graphical representation of plausible hierarchical nanoself-assembly process of MIPAs into bilayer vesicles and its acid driven transformation into hexagonal nanosheets and fibers in a reversible fashion. For legibility of the self-assembly process we denoted simple repeating monomer unit of supramolecular polymer P3. 
molecular switchable MIPAs will lead to a new bottom-up approach for the future optoelectronic applications of nanomaterials.

\section{ASSOCIATED CONTENT}

\section{S Supporting Information}

Complete ref 19; detailed synthetic procedures, schemes, supporting Figures S1-S21, and compound characterization data $\left({ }^{1} \mathrm{H}\right.$ and ${ }^{13} \mathrm{C}$ NMR $)$. This material is available free of charge via the Internet at http://pubs.acs.org.

\section{AUTHOR INFORMATION}

\section{Corresponding Author}

*Tel 8863-5712121 ext 55305; Fax 8863-5724727; e-mail linhc@mail.nctu.edu.tw.

\section{Notes}

The authors declare no competing financial interest.

\section{ACKNOWLEDGMENTS}

We thank the National Science Council of Taiwan (ROC) for financial support of this project through NSC 101-2113-M-009013-MY2 and NSC99-2221-E-009-008-MY2.

\section{REFERENCES}

(1) (a) Collier, C. P.; Mattersteig, G.; Wong, E. W.; Luo, Y.; Beverly, K.; Sampaio, J.; Raymo, F. M.; Stoddart, J. F.; Heath, J. R. Science 2000, 289, 1172-1175. (b) Olson, M. A.; Braunschweig, A. B.; Fang, L.; Ikeda, T.; Klajn, R.; Trabolsi, A.; Wesson, P. J.; Benitez, D.; Mirkin, C. A.; Grazy, B. A.; Stoddart, J. F. Angew. Chem., Int. Ed. 2009, 48, $1792-1797$.

(2) (a) Frampton, M. J.; Anderson, H. L. Angew. Chem., Int. Ed. 2007, 46, 1028-1064. (b) Terao, J.; Tanaka, Y.; Tsuda, S.; Kambe, N.; Taniguchi, M.; Kawai, T.; Saeki, A.; Seki, S. J. Am. Chem. Soc. 2009, 131, 18046-18047. (c) Terao, J. Polym. Chem. 2011, 2, 2444-2452.

(3) (a) Ooya, T.; Choi, H. K.; Yamashita, A.; Yui, N.; Sugaya, Y.; Kano, A.; Maruyama, A.; Akita, H.; Ito, R.; Kogure, K.; Harashima, H. J. Am. Chem. Soc. 2006, 128, 3852-3853. (b) Seo, J. H.; Kakinoki, S.; Inoue, Y.; Yamaoka, T.; Ishihara, K.; Yui, N. J. Am. Chem. Soc. 2013, 135, 5513-5516.

(4) (a) Anderson, H. L.; Anderson, S.; Sanders, J. K. M. Acc. Chem. Res. 1993, 26, 469-475. (b) Werts Michel, P. L.; Boogaard, M. V. D.; Tsivgoulis, G. T.; Hadziioannou, G. Macromolecules 2003, 36, 70047013. (c) Hawker, C. J.; Wooley, K. L. Science 2005, 309, 1200-1205.

(5) (a) Zhu, S. S.; Carroll, P. J.; Swager, T. M. J. Am. Chem. Soc. 1996, 118, 8713-8714. (b) Gong, C.; Ji, Q.; Subramaniam, C.; Gibson, H. W. Macromolecules 1998, 31, 1814-1818. (c) Liu, Y.; Zhao, Y. L.; Zhang, H. Y.; Song, H. B. Angew. Chem., Int. Ed. 2003, 42, 3260-3263. (d) Terao, J.; Tsuda, S.; Tanaka, Y.; Okoshi, K.; Fujihara, T.; Tsuji, Y.; Kambe, N. J. Am. Chem. Soc. 2009, 131, 16004-16005. (e) Lee, Y. G.; Koyam, Y.; Yonekawa, M.; Takata, T. Macromolecules 2010, 43, 4070-4080. (f) Tzeng, B. C.; Wei, S. L.; Chang, T. Y. Chem.-Eur. J. 2012, 18, 16443-16449.

(6) (a) Weidmann, J. L.; Kern, J. M.; Sauvage, J. P.; Geerts, Y.; Muscat, D.; Mullen, K. Chem. Commun. 1996, 1243-1244. (b) Weidmann, J. L.; Kern, J. M.; Sauvage, J. P.; Muscat, D.; Mullins, S.; Kohler, W.; Rosenauer, C.; Radar, H. J.; Martin, K.; Geerts, Y. Chem.-Eur. J. 1999, 5, 1841-1851. (c) Takata, T.; Kawasaki, H.; Kihara, N.; Furusho, Y. Macromolecules 2001, 34, 5449-5456. (d) Kwan, P. H.; Maclachlan, M. J.; Swager, T. M. J. Am. Chem. Soc. 2004, 126, 8638-8639. (e) Taura, D.; Li, S.; Hashidzume, A.; Harada, A. Macromolecules 2010, 43, 1706-1713.

(7) (a) Michels, J. J.; O’Connell, M. J.; Taylor, P. N.; Wilson, J. S.; Cacialli, F.; Anderson, H. L. Chem.-Eur. J. 2003, 9, 6167-6176. (b) Yamaguchi, I.; Kashiwagi, K.; Yamamoto, T. Macromol. Rapid Commun. 2004, 25, 1163-1166.
(8) (a) Fluery, G.; Schlatter, G.; Brochon, C.; Travelet, C.; Lapp, A.; Lindener, P.; Hadziioannou, G. Macromolecules 2007, 40, 535-543. (b) Ren, L.; Ke, F.; Chen, Y.; Liang, D.; Huang, J. Macromolecules 2008, 41, 5295-5300. (c) De Bo, G.; Winter, J. D.; Gerbaux, P.; Fustin, C. A. Angew. Chem., Int. Ed. 2011, 50, 9093-9096.

(9) (a) Ball, P. Nature 2000, 406, 118-120. (b) Scarff, C. A.; Snelling, J. R.; Knust, M. M.; Wilkins, C. L.; Scrives, J. H. J. Am. Chem, Soc. 2012, 134, 9193-9198. (c) Fukuzumi, S.; Ohukubo, K.; D’Souza, F.; Sessler, J. L. Chem. Commun. 2012, 48, 9801-9815.

(10) MacLachlan, M. J.; Rose, A.; Swager, T. M. J. Am. Chem. Soc. 2001, 123, 9180-9181.

(11) Green, J. E.; Choi, J. W.; Boukai, A.; Bunimovich, Y.; Happerin, E. J.; DiIonno, E.; Luo, Y.; Sheriff, B. A.; Xu, K.; Shin, Y. S.; Tseng, R.; Stoddart, J. F.; Heath, J. R. Nature 2007, 445, 414-417.

(12) (a) Wenz, G.; Han, B. H.; Muller, A. Chem. Rev. 2006, 106, 782-817. (b) Harada, A.; Hashidzume, A.; Yamaguchi, H.; Takashima, Y. Chem. Rev. 2009, 109, 5974-6023.

(13) (a) Kim, K. Chem. Soc. Rev. 2002, 31, 96-107. (b) Ko, Y. H.; Kim, E.; Hwang, I.; Kim, K. Chem. Commun. 2007, 1305-1315.

(14) (a) Momcilovic, N.; Clark, P. G.; Boydston, A. J.; Grubbs, R. H. J. Am. Chem. Soc. 2011, 133, 19087-19089. (b) Ji, X.; Yao, Y.; Li, J.; Yan, X.; Huang, F. J. Am. Chem. Soc. 2013, 135, 74-77.

(15) (a) Li, J.; Loh, X. J. Adv. Drug Delivery Rev. 2008, 60, 10001017. (b) Dam, H. H.; Caruso, F. Adv. Mater. 2011, 23, 3026-3029. (c) Yamada, Y.; Nomura, T.; Harashima, H.; Yamashita, A.; Yui, N. Biomaterials 2012, 33, 3952-3958.

(16) Ghadari, M. R.; Granja, J. R.; Buehler, L. K. Nature 1994, 369, 301-304.

(17) Johnson, J.; Fu, N.; Arunkumar, E.; Leevy, W. M.; Gammon, S. T.; Worms, D. P.; Smith, B. D. Angew. Chem., Int. Ed. 2007, 46, 55285531.

(18) Raju, M. V. R.; Lin, H. C. Org. Lett. 2013, 15, 1274-1277.

(19) Frisch, M. J.; et al. Gaussian 09; Gaussian, Inc.: Wallingford, CT, 2009.

(20) Boese, A. D.; Handy, N. C. J. Chem. Phys. 2002, 116, 95599569.

(21) (a) Hamprecht, F. A.; Cohen, A. J.; Tozer, D. J.; Handy, N. C. J. Chem. Phys. 1998, 109, 6264-6271. (b) Boese, A. D.; Doltsinis, N. L.; Handy, N. C.; Sprik, M. J. Chem. Phys. 2000, 112, 1670-1678.

(22) (a) Stewart, J. J. P. J. Comput. Chem. 1989, 10, 209-220. (b) Stewart, J. J. P. J. Comput. Chem. 1989, 10, 221-264.

(23) (a) Hao, Z.; Iqbal, A. Chem. Soc. Rev. 1997, 26, 203-213. (b) Qu, S.; Tian, H. Chem. Commun. 2012, 48, 3039-3051.

(24) Du, C.; Li, C.; Li, W.; Chen, X.; Bo, Z.; Veit, C.; Ma, Z.; Wuerful, U.; Zhu, H.; Hu, W.; Zhang, F. Macromolecules 2011, 44, $7617-7624$.

(25) Zhang, W.; Dichtel, W. R.; Stieg, A. Z.; Benitez, D.; Glimzewski, J. K.; Heath, J. R.; Stoddart, J. F. Proc. Natl. Acad. Sci. U. S. A. 2008, $105,6514-6519$.

(26) (a) Jang, Y. H.; Hwang, S.; Kim, Y. H.; Jang, S. S.; Goddard, W. A. J. Am. Chem. Soc. 2004, 126, 12636-12645. (b) Benitez, D., II; Yoon, T.; Stoddart, J. F.; Goddard, W. A., III J. Am. Chem. Soc. 2008, 130, 14928-14929.

(27) (a) Wu, J.; Gao, C. Macromolecules 2010, 43, 7139-7146. (b) Chen, Y.; Zhang, Y. M.; Liu, Y. Chem. Commun. 2010, 46, 56225633.

(28) (a) Martinez, C. R.; Iverson, B. L. Chem. Sci. 2012, 3, 21912201. (b) Tzeng, B. C.; Wei, S. L.; Chang, T. Y. Chem.-Eur. J. 2012, 18, 5105-5112. (c) Mitra, A.; Panda, D. K.; Carson, L. J.; Saha, S. Chem. Commun. 2013, 49, 4601-4603.

(29) (a) Magonov, S. N. Visualization of polymers at surfaces and interfaces with atomic force microscopy. In Handbook of Surfaces and Interfaces of Materials; Nalwa, H. R., Ed.; Academic: New York, 2001; Vol. 2, pp 393-429. (b) Moulin, J.-F.; Kengne, J. C.; Kshirsagar, R; Cavallani, M.; Biscarini, F.; Leon, S.; Zerbetto, F.; Bottari, G.; Leigh, D. A. J. Am. Chem. Soc. 2006, 128, 526-532.

(30) Fialkowski, M.; Bitner, A.; Grzybowski, B. A. Nat. Mater. 2005, 4, 93-97. 
(31) Hwang, M. J.; Bae, H. S.; Kim, S. J.; Jeong, B. Macromolecules 2004, 37, 8820-8822.

(32) Seo, S. H.; Chang, J. Y.; Tew, G. N. Angew. Chem., Int. Ed. 2006, $45,7526-7530$. 